\title{
ON THE EXISTENCE OF AFFINE LANDAU-GINZBURG PHASES IN GAUGED LINEAR SIGMA MODELS
}

\author{
PATRICK CLARKE ${ }^{\dagger}$ AND JOSH GUFFIN ${ }^{\ddagger}$
}

\begin{abstract}
We prove a simple criterion for the existence of an affine LandauGinzburg point in the Kähler moduli space of a gauged linear sigma model.
\end{abstract}

\section{INTRODUCTION}

A Landau-Ginzburg (LG) point in the Kähler moduli space of a gauged linear sigma model (GLSM) allows one to compute correlation functions which are otherwise inaccessible. In the literature, there is no efficient method for determining the existence of such a point.

GLSMs were introduced in Wit93 as a way of studying correlation functions that compute Gromov-Witten invariants in certain related non-linear sigma models (NLSM). In particular, the low-energy limit on the Higgs branch of a GLSM is a NLSM whose target space is a toric variety $X$ obtained as a symplectic $U(1)^{\rho}$ quotient using the parameters of the GLSM.

For certain classes of GLSMs, one may choose the parameters so that the target space of the low-energy theory is an orbifold $\mathbb{C}^{n} / \Gamma$, with a superpotential whose critical locus lies at the fixed point of the finite abelian group $\Gamma$. Such a setup is called a Landau-Ginzburg theory, and correlators therein are exactly soluble. To find if a given GLSM possesses an LG point, one typically constructs the secondary fan and laboriously checks whether the D- and F-terms for each chamber satisfy the requisite condition. Such a strategy was pursued in $\S 4.2$ of [MRP95, for example.

LG points are also useful in $(0,2)$ GLSMs. These models reduce in the low-energy limit to a NLSM on the same variety $X$, but also depend on a holomorphic vector bundle $\mathcal{V} \rightarrow X$ that is determined by the data of the (0,2) GLSM. Correlation functions in these theories are invariants of $(X, \mathcal{V})$ that generalize the Gromov-Witten invariants of $\left(X, T_{X}\right)$ ADE06. In cases where the bundle $\mathcal{V}$ is a deformation of the tangent bundle, correlators may be computed using a brute-force method based on Čech cohomology [KS06, GK07, or by employing more refined techniques in the $(0,2)$ GLSM MM07. When $\mathcal{V}$ is not a deformation of $T_{X}$, general techniques to 
compute correlators in the NLSM do not exist. However, when the $(0,2)$ GLSM admits an LG phase, correlators may be computed using the methods of Mel09. Our results should admit a generalization to the $(0,2)$ case.

LG theories have also been useful for the computation of Gromov-Witten invariants, following the program initiated by Fan, Jarvis, and Ruan FJR07a, FJR07b. Our results should be especially useful in this setting for producing previously unknown classes of LG models for study (see Remark 3.13).

1.1. The Criterion. Recently, Herbst conjectured Her09] a criterion for the existence of a Landau-Ginzburg point in terms of the charge matrix of the GLSM. The Herbst criterion can be slightly simplified, and we prove that the simplified version is equivalent to the existence of an affine LG point provided the critical locus of the superpotential is compact for some value (and thus all values) of the Kähler parameter. To this end, we also provide a rigorous definition of an affine LG point in a GLSM, and prove the equivalence of symplectic and algebraic quotients for arbitrary GLSMs without regard for smoothness or compactness.

The Herbst Criterion proven herein takes the following form. Consider the charge matrix of the GLSM, $Q$ : if the rank of the GLSM gauge group is $\rho$ and there are $N$ chiral fields, then $Q$ is a $\rho \times N$ matrix of rank $r$. Then an affine LG point exists whenever one can choose $r$ linearly-independent columns so that the other $n:=N-r$ columns lie in the negative cone of the chosen $r$. This setup is a slight generalization of the charge matrices normally considered in the physics literature, where $Q$ is assumed to be full rank. We provide a mathematical setting for $Q$ in our discussion of symplectic quotients - see equation (4).

Herbst originally included the condition that the chosen columns should be unique, in the sense that no column amongst the remaining $n$ is a copy of one of the chosen. However, this is implied by the condition that all others lie in the negative cone. On the other hand, this corollary can be quite useful in showing by hand that a given model does not have an affine LG point. A precise statement of the Herbst Criterion is given in Definition 3.1 .

Heretofore, the main class of toric varieties known to admit affine LG phases were the total spaces of the canonical bundle over compact toric varieties. Our analysis shows that LG phases are extremely common, and easily produced. Indeed, our results provide a way to produce every possible affine LG phase for any GLSM see Remark 3.13 
1.2. Examples. Before proceeding, we present three well-known examples and discuss the application of the criterion to them.

Example 1.1. Consider the canonical bundle of $\mathbb{P}^{m}$. Here, the gauge group is rank 1 and the charges are arranged in a $1 \times(m+2)$ matrix normally written as

$$
Q=\left(\begin{array}{lllll}
1 & 1 & \cdots & 1 & -m-1
\end{array}\right) .
$$

Discarding the first $m+1$ columns due to multiplicity, one is left with the column $(-m-1)$. Since the first $m+1$ columns are in its negative cone, this model will possess an LG point for an appropriate choice of superpotential.

Example 1.2. For the canonical line bundle over the product of rational curves, $K \rightarrow \mathbb{P}^{1} \times \mathbb{P}^{1}$, the gauge group is rank 2 and the charge matrix is normally written

$$
Q=\left(\begin{array}{lllll}
1 & 1 & 0 & 0 & -2 \\
0 & 0 & 1 & 1 & -2
\end{array}\right) .
$$

Examining this matrix, one discards the first four columns since each vector occurs with multiplicity and finds that there is only one independent unique column. Therefore this model cannot have an affine LG point.

Example 1.3. Consider the canonical bundle of the resolved weighted projective space $\widetilde{\mathbb{P}}_{1,1,2,2,2}^{4}$. This model has a rank 2 gauge group and charge matrix

$$
Q=\left(\begin{array}{ccccccc}
0 & 0 & 1 & 1 & 1 & 1 & -4 \\
1 & 1 & 0 & 0 & 0 & -2 & 0
\end{array}\right) .
$$

After discarding the first five columns one is left with the final two, which are linearly independent. It is not hard to check that the first five columns are contained in the negative cone of the last two. One can also use the algorithm outlined in Remark 4.1 to find that the charge matrix row-reduces to

$$
\left(\begin{array}{cc}
1 & -4 \\
-2 & 0
\end{array}\right)^{-1} \cdot Q=\left(\begin{array}{ccccccc}
-\frac{1}{2} & -\frac{1}{2} & 0 & 0 & 0 & 1 & 0 \\
-\frac{1}{8} & -\frac{1}{8} & -\frac{1}{4} & -\frac{1}{4} & -\frac{1}{4} & 0 & 1
\end{array}\right)
$$

so that the first five vectors clearly lie in the negative cone. Thus, for an appropriate choice of superpotential this model will possess an LG point.

Acknowledgments. We thank Manfred Herbst for helpful comments on an early draft of this manuscript, Jacques Distler for useful conversations, and the organizers of the 2009 conference on (0,2) Mirror Symmetry and Quantum Sheaf Cohomology at the Max-Planck-Institut für Gravitationsphysik where this work began. This material is based upon work supported by the National Science Foundation under DMS Grant No. 0636606 and 0703643. 


\section{Physical Exposition}

We first explain the physical origins of LG points in the GLSM. We will always assume that we are dealing with a GLSM featuring a gauge group of rank $\rho$ and $N$ chiral bosons, whose low-energy theory describes a toric variety $X$ for appropriate values of its Kähler parameters.

To determine the low-energy theory, one imposes a system of constraints arising as the classical equations of motion of the GLSM Lagrangian on the chiral bosons in the theory. In terms of the charge matrix $Q$, chiral bosons $\phi^{i}$, and Kähler parameters $r^{a}$, one has $r$ equations

$$
\sum_{i=1}^{N} Q_{i}^{a}\left|\phi^{i}\right|^{2}-r^{a}=0 \quad \text { for } 1 \leq a \leq r .
$$

Here $r$ is the rank of $Q$. For the purposes of this paper, we will simply call the equations above $D$-terms.

The D-terms specify the construction of a toric variety $X$ as a $U(1)^{\rho}$ quotient, as in equation (77). The low-energy theory is also dependent on another class of terms arising from a torus-invariant holomorphic function $W: \mathbb{C}^{N} \rightarrow \mathbb{C}$ called the superpotential. Several terms in the Lagrangian involve this function, but we will concentrate on one set in particular whose vanishing is required for supersymmetric vacua:

$$
\sum_{i=1}^{N}\left|\frac{\partial W}{\partial \phi^{i}}\right|^{2}=0 .
$$

For the purposes of this paper, these will be called F-terms.

Let $\mathcal{V} \rightarrow Y$ be a vector bundle of rank $k$ over a compact toric variety $Y$, whose total space is the $n$-dimensional toric variety $X$. Let $Z \subset Y$ be the smooth vanishing locus of a holomorphic section $f: Y \rightarrow \mathcal{V}^{\vee}$. For certain values of the Kähler parameters those in the Kähler cone - such a compact complete intersection is realized as the target space in the low-energy theory of a GLSM whose superpotential is

$$
W=\sum_{\alpha=1}^{k} p_{\alpha} f^{\alpha}(\phi),
$$

where $p_{\alpha}$ are a subset of the bosonic fields associated with fiber coordinates on $\mathcal{V}$ and the $\phi$ are fields associated with the base $Y$. Since the zero locus of $f$ is assumed to be smooth, the critical points of $W$ are precisely the vanishing locus of $f$, lying 
within the zero section of the bundle.

The model possesses an LG point if there is some value of the Kähler parameters (taken to lie deep in the interior of a top-dimensional cone of the secondary fan) such that solving the D- and F-terms requires that precisely $r$ of the bosons get a vacuum expectation value (VEV), while the others vanish. Additionally, one requires that the low-energy superpotential in this phase has a single degenerate critical point. In other words, an LG point is a choice of Kähler parameters such that the low-energy physics is described by a quantum field theory whose bosonic fields are valued in a vector space (in particular $\mathbb{C}^{n}$, or more generally its quotient by a finite abelian group), governed by a superpotential, and whose space of vacua consists of a single point. One must take the parameters to be deep inside a cone to avoid subtleties from quantum corrections.

\section{Mathematical Exposition}

Dividing out the $U(1)^{\rho}$ symmetries of a GLSM with specified D-terms leads to a toric variety which, along with the superpotential, governs its physics. The toric variety is obtained as a symplectic quotient at an appropriate value of the moment map (the $r^{a}$ in 2).

It is well-known that if the value of the moment map is regular and the quotient is compact, then it is a projective toric manifold Aud00. It is not hard to imagine, though less well-known, that the quotient is a quasi-projective variety at any value of the moment map, regardless of regularity or compactness. We provide a proof of this fact in $\$ 3.1$.

The group of characters on the quotient, $M \cong \mathbb{Z}^{\oplus n}$, fits into an exact sequence

$$
0 \rightarrow M \stackrel{A}{\longrightarrow}\left(\mathfrak{u}(1)_{\mathbb{Z}}^{N}\right)^{*} \stackrel{Q}{\longrightarrow}\left(\mathfrak{u}(1)_{\mathbb{Z}}^{\rho}\right)^{*} .
$$

Here $\mathfrak{u}(1)^{N}$ is the Lie algebra of a maximal torus in $\operatorname{Aut}\left(\mathbb{C}^{N}\right)$ commuting with the action of $\mathfrak{u}(1)^{\rho}$ and $\mathfrak{u}(1)_{\mathbb{Z}}$ denotes the kernel of the exponential map. The $\mathbb{R}$-linear maps obtained from these by $\otimes_{\mathbb{Z}} \mathbb{R}$ are denoted by the same name.

As in equation (6) below, $Q^{t}$ specifies the a linear action of $\left(\mathbb{C}^{*}\right)^{\rho}$ on $\mathbb{C}^{N}$ and $A$ is the kernel of $Q$. Physically, $Q$ is the matrix of charges appearing in the D-terms (2) of the GLSM. The Lie algebra $\mathfrak{u}(1)^{N}$ has a canonical set, $C=\left\{\partial_{\psi_{1}} \cdots \partial_{\psi_{N}}\right\}$, corresponding to coordinates $z_{j}$ with $\arg z_{j}=2 \pi \psi_{j}$. 
Definition 3.1. The map $Q$ in equation (4) satisfies the Herbst Criterion if there exists a subset $\left\{h_{1}, \cdots, h_{r}\right\}$ of $\left\{\partial_{\psi_{1}}, \cdots, \partial_{\psi_{N}}\right\}$ such that $\left\{Q h_{1}, \cdots, Q h_{r}\right\}$ are linearly independent and

$$
Q C \backslash\left\{Q h_{1}, \cdots, Q h_{r}\right\} \subset \mathbb{R}_{\leq 0} Q h_{1}+\cdots+\mathbb{R}_{\leq 0} Q h_{r} .
$$

In particular, isomorphisms $\left(\mathfrak{u}(1)^{\rho}\right)^{*} \cong \mathbb{R}^{\rho}=\mathbb{Z}^{\rho} \otimes_{\mathbb{Z}} \mathbb{R}$ and $\left(\mathfrak{u}(1)^{N}\right)^{*} \cong \mathbb{R}^{N}=$ $\mathbb{Z}^{N} \otimes_{\mathbb{Z}} \mathbb{R}$ give an integer matrix expression for $Q$. The criterion is satisfied if there exists an reordering of the basis of $\mathbb{R}^{N}$ and a rational change of basis for $\mathbb{R}^{\rho}$ so that

$$
Q=\left(\begin{array}{cc}
\mathbb{1}_{r \times r} & \mathbf{n}_{r \times n} \\
0 & 0
\end{array}\right)
$$

with the entries of $\mathbf{n}$ non-positive rational numbers. We will prove the equivalence of the Herbst Criterion to the existence of an affine quotient in $\$ 3.2$.

3.1. Toric Quotients. The most important consequence of the equality of symplectic and algebraic quotients is that we can read off the algebro-geometric description of the quotient from its image under the moment map. This image, known as the moment polyhedral set, is defined by a finite family of inequalities.

Later, we rely on the geometry of the polyhedral set to understand when quotient is unchanged for small changes in the Kähler parameter.

Every linear action of an algebraic torus on an $N$-dimensional complex vector space $V,\left(\mathbb{C}^{*}\right)^{\rho} \circlearrowright V$, may be unitarily diagonalized so that for any $\vec{\lambda} \in\left(\mathbb{C}^{*}\right)^{\rho}, \vec{z} \in \mathbb{C}^{N}$, and integers $Q_{i j}^{t}$ for $1 \leq i \leq N, 1 \leq j \leq \rho$,

$$
\vec{\lambda} \star \vec{z}=\left(\lambda_{1}^{Q_{11}^{t}} \cdots \lambda_{\rho}^{Q_{1 \rho}^{t}} z_{1}, \cdots, \lambda_{1}^{Q_{N 1}^{t}} \cdots \lambda_{\rho}^{Q_{N \rho}^{t}} z_{N}\right) .
$$

The standard symplectic form $\omega=\frac{\sqrt{-1}}{2} \sum_{i} d z^{i} \wedge d \bar{z}^{i}$ and the standard action of $U(1)^{N}$ on $\mathbb{C}^{N}$ define a moment map

$$
\mu: \mathbb{C}^{N} \rightarrow \mathbb{R}^{N}=\left(\mathfrak{u}(1)^{N}\right)^{*} .
$$

In coordinates, $\mu$ is given by $\vec{z} \mapsto \frac{1}{2}\left(\left|z_{i}\right|^{2}\right)_{i}$.

The moment map for the action of $U(1)^{\rho} \subset\left(\mathbb{C}^{*}\right)^{\rho}$ on $\left(\mathbb{C}^{N}, \omega\right)$ is given by the composition

$$
\mu_{Q}=Q \circ \mu: \mathbb{C}^{N} \rightarrow \mathbb{R}^{\rho}=\left(\mathfrak{u}(1)^{\rho}\right)^{*} .
$$

Because of D-term contributions (2) to the Lagrangian, the physical action is stationary for maps $\Sigma \rightarrow \mu_{Q}^{-1}(s)$ for a choice of $s \in \mathbb{R}^{\rho}$. If $s$ is not in the image of 
$Q, X$ is empty. Otherwise, $s$ corresponds to $r^{a}$ in the D-terms. These maps are taken up to the action of $U(1)^{\rho}$ on $\mu_{Q}^{-1}(s)$. Thus, it is equivalent to consider maps $\Sigma \rightarrow X$, where

$$
X=\mu_{Q}^{-1}(s) / U(1)^{\rho} .
$$

$X$ naturally carries the structure of a toric variety and can be written as a geometric quotient as follows. Define $E$ to be the complement of $\left(\mathbb{C}^{*}\right)^{N} \star \mu_{Q}^{-1}(s)$ in $\mathbb{C}^{N}$, and consider

$$
\mathbb{X}=\left(\mathbb{C}^{N} \backslash E\right) /\left(\mathbb{C}^{*}\right)^{\rho} .
$$

Since the $U(1)^{\rho}$ orbits are contained in the $\left(\mathbb{C}^{*}\right)^{\rho}$ orbits, there is a natural map $X \rightarrow \mathbb{X}$. We will show that this map is an isomorphism. We first check that it is an injection by showing that the orbits $\mathbb{R}_{+}^{r} \star \vec{z}$ for $\vec{z} \in \mu_{Q}^{-1}(s)$ are disjoint. This is accomplished by showing that $\mu_{Q}$ restricted to such an orbit is injective.

Lemma 3.2. $X \rightarrow \mathbb{X}$ is injective

Proof. The action of $\mathbb{R}_{+}^{\rho} \subset\left(\mathbb{C}^{*}\right)^{\rho}$ on $\mathbb{C}^{N}$ induces an action of $\mathbb{R}_{+}^{\rho}$ on $\mathbb{R}_{\geq 0}^{N}$ defined by $\vec{\lambda} \star \mu(\vec{z})=\mu(\vec{\lambda} \star \vec{z})$. It is easy to see that this is independent of the choice of $\vec{z}$, as different choices are given by the action of $U(1)^{N}$. Directly, the action $\mathbb{R}_{+}^{\rho}$ on $\mathbb{R}_{\geq 0}^{N}$ is given by

$$
\vec{\lambda} \star \vec{q}=\vec{\lambda}^{Q^{t}} \vec{q}=\operatorname{diag}\left(\lambda^{Q^{t}}, \cdots \lambda^{Q^{t}{ }_{N}}\right) \vec{q}
$$

where $\lambda^{Q^{t}{ }_{i}}=\lambda_{1}^{Q^{t}}{ }_{i 1} \cdots \lambda_{\rho}^{Q^{t}{ }_{i \rho}}$.

We would like to show that the action of $\mathbb{R}_{+}^{r}$ on $\mathbb{C}^{N}$ changes the value of $\mu_{Q}$. It suffices to show that $\mathbb{R}_{+}^{r}$ on $\mathbb{R}_{\geq 0}^{N}$ changes the value of $q$. Concretely, we wish to show $Q\left(\mathbb{1}-\vec{\lambda}^{Q^{t}}\right) \vec{q}=0$ has no solutions except $\vec{q}=0$.

If we denote the kernel of $Q$ by $A$ as in equation (4), then it is equivalent to show

$$
\left(\mathbb{1}-\vec{\lambda}^{Q^{t}}\right) \mathbb{R}_{\geq 0}^{N} \cap \operatorname{Im} A=\{0\} .
$$

Because both $1-x$ and $-\log (x)$ are positive/negative/zero on the same set we have

$$
\left(\mathbb{1}-\vec{\lambda}^{Q^{t}}\right) \mathbb{R}_{\geq 0}^{N}=-\log \left(\vec{\lambda}^{Q^{t}}\right) \mathbb{R}_{\geq 0}^{N}=-\operatorname{diag}\left(Q^{t} \log (\vec{\lambda})\right) \mathbb{R}_{\geq 0}^{N} .
$$

An element of $-\operatorname{diag}\left(Q^{t} \log (\vec{\lambda})\right) \mathbb{R}_{\geq 0}^{N}$ is non-zero if and only if its dot product the vector

$$
-\operatorname{diag}\left(Q^{t} \log (\vec{\lambda})\right)\left[\begin{array}{c}
1 \\
\vdots \\
1
\end{array}\right]=-Q^{t} \log (\vec{\lambda})
$$


is non-zero. Finally, observe that the dot product for any $v \in M_{\mathbb{R}}$ is given by

$$
-Q^{t} \log (\vec{\lambda}) \cdot A v=-\log (\vec{\lambda})^{t} Q A v=0 .
$$

Lemma 3.3. $X \rightarrow \mathbb{X}$ is surjective.

Proof. First notice that Lemma 3.2 guarantees $X=\left(\left(\mathbb{C}^{*}\right)^{\rho} \star \mu_{Q}^{-1}(s)\right) /\left(\mathbb{C}^{*}\right)^{\rho}$. So, we need to check that

$$
\left(\mathbb{C}^{*}\right)^{N} \star \mu_{Q}^{-1}(s)=\left(\mathbb{C}^{*}\right)^{\rho} \star \mu_{Q}^{-1}(s) .
$$

We will show by construction that given any element $p \in\left(\mathbb{C}^{*}\right)^{N} \star \mu_{Q}^{-1}(s)$ there exists an element $h \in\left(\mathbb{C}^{*}\right)^{\rho}$ such that $\mu_{Q}(h \star p)=s$. Choosing $g$ such that $\mu_{Q}(g \star p)=s$, $h$ will be constructed by lifting the curve $\gamma(t)=\mu_{Q}(\exp (t \ln g) \star p) \subset \mathbb{R}^{\rho}$ to a curve in $\left(\mathbb{C}^{*}\right)^{\rho}$.

Denote the Lie algebra of $\left(\mathbb{C}^{*}\right)^{N}$ by $\mathfrak{g}$ and the Lie algebra of $\left(\mathbb{C}^{*}\right)^{\rho}$ by $\mathfrak{h}$. Then, given a curve $\eta:[0,1]_{t} \rightarrow \mathfrak{g}$, we obtain a curve $P \exp (\eta):[0,1]_{t} \rightarrow\left(\mathbb{C}^{*}\right)^{N}$ defined by $\frac{d}{d t} P \exp (\eta)=\eta$. Furthermore if $\eta$ lies in $\mathfrak{h}$ then the resulting curve is in $\left(\mathbb{C}^{*}\right)^{\rho}$. To be clear, this notation is with respect to the trivialization by right-invariant vector fields: $T_{e}\left(\mathbb{C}^{*}\right)^{N}=\mathfrak{g}$.

The typical fibre of the tangent bundle of $\left(\mathbb{C}^{*}\right)^{N} \times \mathbb{C}^{N}$ is $\mathfrak{g} \oplus \mathbb{C}^{N}$. Consider the differential $d\left(\mu_{Q} \circ \star\right)$ of the composition of $\star$ with $\mu_{Q}: \mathbb{C}^{N} \rightarrow \mathbb{R}^{\rho}$. Restricting to $\mathfrak{g}$ induces a map from Lie algebra of $\left(\mathbb{C}^{*}\right)^{N}$ to the tangent space of $\mathbb{R}^{\rho}$. Given a curve $\gamma:[0,1] \rightarrow \mathbb{R}^{\rho}$ such that $\gamma^{\prime}(t)$ is in the image of $\mathfrak{g}$ under $d\left(\mu_{Q} \circ \star\right)$, denote the lifted curve by $\hat{\gamma}:[0,1] \rightarrow \mathfrak{g}$. Observe that it has the property that $\mu_{Q}\left(P \exp (\hat{\gamma}(t)) \star l_{0}\right)=\gamma(t)$ for any choice $l_{0} \in \mu_{Q}^{-1}(\gamma(0))$.

Now consider the curve $\gamma(t)=\mu_{Q}(\exp (t \ln g) \star p)$. Observe that $\gamma(0)=\mu_{Q}(p)$ and $\gamma(1)=s$. The proof then depends on the existence of a lift of $\gamma^{\prime}, \eta:[0,1] \rightarrow$ $\mathfrak{h} \subset \mathfrak{g}$. Once we have this lift, we can take $h=P \exp (\eta(1))$. By construction, $\gamma^{\prime} \subset d\left(\mu_{Q} \circ \star\right)(\mathfrak{g})$ since $\hat{\gamma}(t)=t \ln g$ is a lift to $\mathfrak{g}$. We will prove the existence of $\eta$ by showing $d\left(\mu_{Q} \circ \star\right)(\mathfrak{g})=d\left(\mu_{Q} \circ \star\right)(\mathfrak{h})$.

The differential $d\left(\mu_{Q} \circ \star\right)$ annihilates $\mathfrak{u}(1)^{N}$. So, we can restrict our attention to its evaluation on $\mathbb{R}_{+}^{N}$. As before, the action is diagonal and so the rank of the differential at $\vec{q} \in \mathbb{R}_{\geq 0}$ equals the dimension of the smallest coordinate subspace containing $\vec{q}$ - that is, the number of non-zero entries of $\vec{q}$. Note that it suffices to check when $\vec{q}$ has all non-zero entries, as the appearance of a zero-entry is the same 
as replacing $N$ with $N-1$.

As in equation (8), the action of $\mathbb{R}_{+}^{\rho}$ is given by the product $\vec{\lambda}^{Q^{t}} \vec{q}$. The differential of the action is

$$
d\left(\vec{\lambda}^{Q^{t}}\right) \cdot \vec{q}=\operatorname{diag}\left(\sum_{j=1}^{\rho} \lambda^{Q_{i}^{t}} Q_{i j}^{t} d \log \lambda_{j}\right)_{i} \cdot \vec{q} .
$$

The Jacobian can be written $\operatorname{diag}\left(q_{i} \lambda^{Q_{i}^{t}}\right) Q^{t}$, and it follows that the Jacobian of $\mu_{Q} \circ \star$ is given by $Q \operatorname{diag}\left(q_{i} \lambda^{Q_{i}^{t}}\right) Q^{t}$. If we set $f^{2}=\operatorname{diag}\left(q_{i} \lambda_{i}^{Q^{t}}\right)$ for a diagonal square matrix $f$, then we can write the Jacobian as

$$
\left(f Q^{t}\right)^{t}\left(f Q^{t}\right)
$$

The rank of $f Q^{t}$ is the rank of $Q^{t}$, and it is not too difficult to check that any matrix of the form $L^{t} L$ has the same rank as $L$. It is also easy to check that the rank of $d\left(\mu_{Q} \circ \star\right)$ also equals the rank of $Q^{t}$, so we are done.

One consequence of these proofs is that if $\operatorname{Stab}_{U(1)^{\rho}}(\vec{z})$ is isomorphic to $T \times U(1)^{\ell}$, for some torsion group $T$, then $\operatorname{Stab}_{\left(\mathbb{C}^{*}\right)^{\rho}}(\vec{\lambda} \star \vec{z})$ is isomorphic to $T \times\left(\mathbb{C}^{*}\right)^{\ell}$ for any $\vec{\lambda} \in\left(\mathbb{C}^{*}\right)^{\rho}$.

As with usual symplectic quotients (no regularity assumption here), the map $\mu$ restricted to $\mu_{Q}^{-1}(s)$ descends to a map $\mu_{X}: X \rightarrow \mathbb{R}^{N} \cdot \mu_{X}(X)$ lies in the affine translation of $\operatorname{Im} A$ over $s$, so we regard it as a map

$$
\mu_{X}: X \rightarrow \operatorname{Im} A \cong M_{\mathbb{R}} .
$$

Here $M_{\mathbb{R}}=M \otimes_{\mathbb{Z}} \mathbb{R}$.

3.2. The Herbst Criterion and affine Landau-Ginzburg points. The vector space im $Q \cong \mathbb{R}^{r} \subset\left(\mathfrak{u}(1)^{\rho}\right)^{*}$ may be decomposed into regions fitting together as a polyhedral fan known as the secondary fan BFS90. The level sets of $\mu_{Q}$ over the relative interior of its top-dimensional cones define isomorphic toric varieties.

Furthermore, given two adjacent top-dimensional cones and corresponding varieties, the quotient construction in equation (7) applied to the codimension-one cone separating them induces a proper birational transformation between the two varieties. See $\S 3.4$ of [CK00] for a nice exposition.

Given a point $s \in \mathbb{R}^{r}$, one constructs the corresponding polytope by first choosing a lift $\widetilde{s} \in \mathbb{R}^{N}$ such that $Q(\widetilde{s})=s$. The polytope is defined to be

$$
P_{s}:=\left\{m \in M_{\mathbb{R}} \mid A(m)+\tilde{s} \geq 0\right\} .
$$


The constraints in the definition of $P_{s}$ are easily reinterpreted as a collection of $N$ half-spaces in $M_{\mathbb{R}}$ whose inward normal vectors are the rows of $A$. Furthermore, one can show that the image of the polytope under the map $m \mapsto A(m)+\widetilde{s}$ is exactly $\mu_{X}(X)$. For this reason, $P_{s}$ is known as the moment polyhedral set of $X$ at level $s$.

Definition 3.4. The relative interior of a top-dimensional cone of the secondary fan is called a phase of the associated GLSM.

Definition 3.5. A point in the secondary fan is stable if it is contained in the relative interior of a top-dimensional cone.

Definition 3.6. A stable point $s \in \mathbb{R}^{r}$ is affine if the polytope $P_{s}$ is a topdimensional simplicial cone in $M_{\mathbb{R}}$.

The nomenclature affine is justified, since via standard construction Ful93, vertices in the polytope correspond to affine open sets that are glued together using the data of higher dimension faces. As there is one vertex in a polyhedral cone there is only one open set. In $\$ 3.3$ we will show that in fact the quotient is $\mathbb{C}^{n} / \Gamma$ for $\Gamma$ a finite abelian group.

Lemma 3.7. The map $Q$ satisfies the Herbst Criterion iff there is an affine stable point in its image.

Proof. Consider a charge matrix $Q$ satisfying the Herbst Criterion - it may be written in the form (5). Since $A$ is full rank, there is a basis such that

$$
A=\left(\begin{array}{c}
\mathbf{N}_{r \times n} \\
\mathbb{1}_{n \times n}
\end{array}\right) .
$$

Furthermore, the sequence (44) implies that $\mathbf{n}_{r \times n}=-\mathbf{N}_{r \times n}$.

We now use these facts to construct a simplicial polytope. Select a point $\sigma$ in the positive orthant of $\mathbb{R}^{r}$ (positive in the basis chosen so that $Q$ is as in equation (5)). One may then select a lift $\tilde{\sigma} \in \mathbb{R}^{N}$ whose first $r$ entries are $\sigma$ and whose final $n$ entries are zero.

The resulting half-spaces are of two types: those arising the first $r$ rows of $A$, and those arising from the last $n$ rows. Those defined by the first $r$ rows are of the form

$$
H_{i}^{+}=\left\{m \in M_{\mathbb{R}} \mid \mathbf{N}_{i} \cdot m \geq-\sigma_{i}\right\}
$$

while those defined by the last $n$ rows take the form

$$
H_{j}^{+}=\left\{m \in M_{\mathbb{R}} \mid m_{j} \geq 0\right\} .
$$


Half-spaces defined by the last rows pick out the positive orthant of $M_{\mathbb{R}}$, and since the entries of $\mathbf{N}$ are all non-negative, any $m \in M_{\mathbb{R}}^{+}$will satisfy the inequality (11). Thus, the polytope consists of the positive orthant, which is a top-dimensional simplicial cone in $M_{\mathbb{R}}$. Since $\sigma$ may be taken to be any element in the positive orthant, one may choose it to be stable.

To prove the converse, let $P \subset M_{\mathbb{R}} \cong \mathbb{R}^{n}$ be a top-dimensional simplicial cone defined by $N$ half-spaces. We first show by contradiction that the inward-pointing normal vectors of all $N$ half-spaces are contained in $P$.

By translations, we can take the apex of the cone $P$ to be the origin in $M_{\mathbb{R}}$. Let $\left\{e_{1}, \cdots, e_{n}\right\}$ generate the rays of $P$, so that $P=\operatorname{Cone}\left(e_{1}, \cdots, e_{n}\right)$. Define $\langle$, so these vectors are orthonormal. Let $H^{+}$be a half-space with normal $\zeta \notin P$ : by definition, we have

$$
H^{+}:=\left\{m \in M_{\mathbb{R}} \mid\langle m, \zeta\rangle \geq-a\right\} .
$$

Note that if $a<0, H^{+}$does not contain the origin, so that $a \geq 0$. Furthermore, stability implies that $a>0$.

Since $\zeta \notin \operatorname{Cone}\left(e_{1}, \cdots, e_{n}\right)$, there exists an $e_{j}$ with $\left\langle e_{j}, \zeta\right\rangle=\beta<0$. Then for all $\alpha>|a / \beta|$,

$$
\left\langle\alpha e_{j}, \zeta\right\rangle=\alpha \beta<-|a| .
$$

Therefore $\alpha e_{j}$ is not in $\operatorname{Cone}\left(e_{1}, \cdots, e_{n}\right)$, a contradiction.

Since all the inward normals are positive, a basis for $A$ exists (the $e_{j}$ ) such that (10) holds with all entries of $\mathbf{N}$ positive - exactly the Herbst Criterion.

Definition 3.8. An affine Landau-Ginzburg point of a GLSM is an affine stable point such that $W: X \rightarrow \mathbb{C}$ has an isolated critical point. The phase in which an affine LG point lies is known as an affine Landau-Ginzburg phase.

Hereafter, we will refer to an affine LG point (phase) as an LG point (phase). As mentioned earlier, in an LG phase $X \cong \mathbb{C}^{n} / \Gamma$ for a finite group $\Gamma \subset U(1)^{n}$. We shall explain the origin of $\Gamma$ and how to compute it in $\$ 3.3$. Also, note that if the critical locus of $W$ is compact at an affine stable point then it is zero-dimensional, and a "nearby" LG phase may be found by modifying the coefficients of the monomials in $W$ so that its critical locus contracts to a single point. In fact, compactness of the critical locus is independent of phase, as the following lemma shows.

Lemma 3.9. Consider a GLSM with secondary fan $\Sigma$ and two top-dimensional cones $\sigma, \sigma^{\prime} \subset \Sigma$ and corresponding toric varieties $X, X^{\prime}$. If $W$ is a function on $X$ 
with compact critical locus, the induced function $W^{\prime}$ on $X^{\prime}$ has compact critical locus as well.

Proof. $X$ and $X^{\prime}$ are related by a proper birational transformation over the toric variety defined by $\sigma^{\prime \prime}=\sigma \cap \sigma^{\prime}$. The critical loci are related by strict transform, because $W$ and $W^{\prime}$ factor through $W^{\prime \prime}$. Such operations do not effect the compactness or non-compactness of a set.

Remark 3.10. If $W: \mathbb{C}^{N} \rightarrow \mathbb{C}$ is a $\left(\mathbb{C}^{*}\right)^{\rho}$-invariant function whose critical locus is compact in some phase, its critical locus is compact in every phase.

Theorem 3.11. The Herbst Criterion together with a $\left(\mathbb{C}^{*}\right)^{\rho}$-invariant function on $\mathbb{C}^{N}$ whose critical locus is compact after quotienting (in any/every phase) is equivalent to the existence of an affine Landau-Ginzburg point.

Proof. Immediate, by Lemmas 3.7 and 3.9 .

For convenience, let us define the cone of an $r \times n$ integer matrix as the cone over the convex hull of its columns, thought of as $n$ elements of $\mathbb{R}^{r}$.

Definition 3.12. Let $T: \mathbb{Z}^{r} \rightarrow \mathbb{Z}^{n}$ and fix a basis for $\mathbb{Z}^{r}$. Then

$$
\operatorname{Cone}(T):=\left\{\nu \in \mathbb{R}^{n}=\mathbb{Z}^{n} \otimes_{\mathbb{Z}} \mathbb{R} \mid \nu=T(\rho) \text { for } \rho \in \mathbb{R}_{\geq 0}^{r}\right\} .
$$

Remark 3.13. Theorem 3.11 shows that GLSMs admitting an affine LandauGinzburg phase are both extremely common and easily produced. One may be found by simply choosing an arbitrary $r \times r$ integer matrix $R$ with non-zero determinant and selecting a finite set $S \subset \operatorname{Cone}(-R) \cap \mathbb{Z}^{r}$. Then the matrix whose entries are $R$ and $S$, as in equation (12) below, satisfies the Herbst criterion.

3.3. Orbifold Structure of the Landau-Ginzburg Phase. Of great importance to the physics of the LG model is the finite group $\Gamma$. It determines the twisted sector of the model, which controls much of the non-trivial dynamics. As we now show, this group is inherited from the $U(1)^{r}$ action in the GLSM as the stabilizer of certain coordinates on $\mathbb{C}^{N}$.

Consider a charge matrix $Q$ satisfying the Herbst Criterion, assumed for simplicity to be a full-rank matrix. Order the columns of $Q$ so that it takes the form

$$
Q=\left(\begin{array}{ll}
R & S
\end{array}\right),
$$

with $R$ an $r \times r$ integer matrix with non-zero determinant such that $R^{-1} \cdot Q$ is of the form given in equation (5). 
Choose $s$ to lie in the relative interior of Cone $(R) \subset \mathbb{R}^{r}$ so that the polytope is a simplicial cone, and consider the quotient $X$ as the algebraic quotient $\left(\mathbb{C}^{N} \backslash E\right) /\left(\mathbb{C}^{*}\right)^{r}$. Since the polytope is a cone, the excluded set is the union of coordinate hyperplanes in $\mathbb{C}^{N}$ corresponding to the half-spaces that do not define codimension-one faces of the cone.

The action of the torus $\left(\mathbb{C}^{*}\right)^{r}$ on $\operatorname{Spec} \mathbb{C}\left[x_{1}^{ \pm 1}, \cdots, x_{r}^{ \pm 1}, y_{1}, \cdots, y_{n}\right]=\left(\mathbb{C}^{N} \backslash E\right)$ is then

$$
\begin{aligned}
\mathbb{C}\left[x_{1}^{ \pm 1}, \cdots, x_{r}^{ \pm 1}, y_{1}, \cdots, y_{n}\right] & \rightarrow \mathbb{C}\left[z_{1}^{ \pm 1}, \cdots, z_{r}^{ \pm 1}, x_{1}^{ \pm 1}, \cdots, x_{r}^{ \pm 1}, y_{1}, \cdots, y_{n}\right] \\
x_{i} & \mapsto x_{i} z^{R_{i}^{t}} \\
y_{j} & \mapsto y_{j} z^{S_{j}^{t}} .
\end{aligned}
$$

Here, we have set $z^{R_{i}^{t}}=\prod_{k} z_{k}^{R_{i k}^{t}}$ and $z^{S_{j}^{t}}=\prod_{k} z_{k}^{S_{j k}^{t}}$ as in equation (6). Define $\Gamma \subset\left(\mathbb{C}^{*}\right)^{r}$ to be the stabilizer of the $x$ 's under this action:

$$
\Gamma:=\operatorname{Spec} \frac{\mathbb{C}\left[z_{1}^{ \pm 1}, \cdots, z_{r}^{ \pm 1}\right]}{\left\langle z^{R_{1}^{t}}-1, \cdots, z^{R_{r}^{t}}-1\right\rangle} \subset\left(\mathbb{C}^{*}\right)^{r} .
$$

Lemma 3.14. Consider $R^{t}: \mathbb{Z}^{r} \rightarrow \mathbb{Z}^{r}$ as a morphism of abelian groups. Then $\Gamma \cong \operatorname{cok} R^{t}$ is a finite abelian group.

Proof. Note that $\Gamma \subset U(1)^{r} \subset\left(\mathbb{C}^{*}\right)^{r}$ iff for all $z \in \Gamma, 1 / z \in \Gamma$. By inverting the relation $z^{R^{t}}=1$, we have $1=1 / z^{R_{j}^{t}}=\prod_{k=1}^{r}\left(1 / z_{k}\right)^{R_{k j}}$ so $\Gamma \subset U(1)^{r}$. Now, consider the following commutative diagram of abelian groups with exact rows:

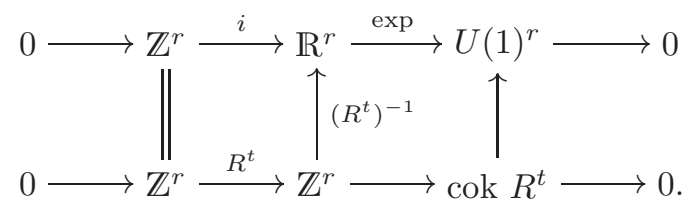

Let $z \in \operatorname{cok} R^{t}$, and $\widetilde{z} \in \mathbb{Z}^{r}$ a lift of $z$. Then, composing the lift with $\left(R^{t}\right)^{-1}$ and $\exp$ yields $\widetilde{z} \mapsto \exp \left(2 \pi i\left(R^{t}\right)^{-1} \cdot \widetilde{z}\right)$, which satisfies

$$
\exp \left(2 \pi i\left(R^{t}\right)^{-1} \widetilde{z}\right)^{R^{t}}=e^{2 \pi i \widetilde{z}}=1 .
$$

Thus cok $R^{t} \subset \Gamma$.

Let $\gamma \in \Gamma$ be a non-trivial element, and choose a lift $\widetilde{\gamma} \in \mathbb{R}^{r}$. Since $\gamma^{R^{t}}=1$, $\left(R^{t}\right) \cdot \widetilde{\gamma} \subset \mathbb{Z}^{r}$, so $\widetilde{\gamma}$ maps to $\mathbb{Z}^{r}$ in the bottom row. Furthermore, $\left(R^{t}\right)^{-1} \widetilde{\gamma}$ is not in the image of $\mathbb{Z}^{r}$, since otherwise $\gamma=1$. Thus, it lies in the cokernel and $\Gamma \subset \operatorname{cok} R^{t}$.

In both cases, lift independence follows from usual diagram chasing. Since $R$ is full-rank, we have that cok $R^{t} \cong \Gamma$ is a finite abelian group. 
It will also be important for us to know the precise form of $\Gamma$.

Proposition 3.15. Let $D$ be the Smith normal form of $R, D=\mathrm{URV}$ with $\mathrm{U}$ and $\mathrm{V}$ invertible over $\mathbb{Z}$, and denote its diagonal entries by $d_{i}$ for $1 \leq i \leq r$. Then

$$
\Gamma \cong \mathbb{Z}_{d_{1}} \times \cdots \times \mathbb{Z}_{d_{r}} \cong \operatorname{Spec} \frac{\mathbb{C}\left[\zeta_{1}^{ \pm 1}, \cdots, \zeta_{r}^{ \pm 1}\right]}{\left\langle\zeta_{1}^{d_{1}}-1, \cdots, \zeta_{r}^{d_{r}}-1\right\rangle}
$$

Proof. By Lemma 3.14 $\Gamma \cong$ cok $R^{t}$. Transposing the expression of the Smith normal form above, we obtain a commutative diagram of abelian groups with exact rows:

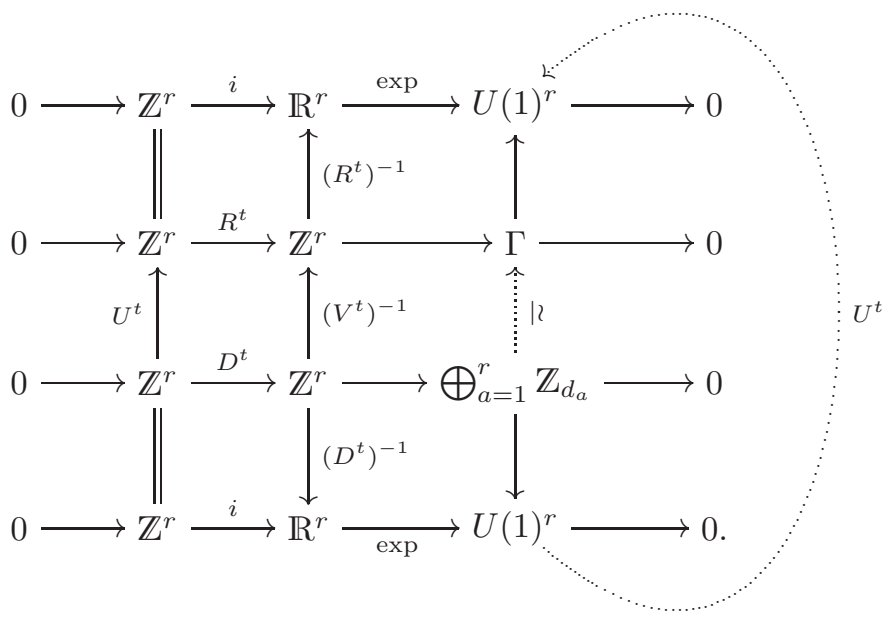

The induced morphism between $U(1)^{r}$ and $U(1)^{r}$ obtains by first lifting and then composing the vertical morphisms in the center columns, $U^{t}=\left(R^{t}\right)^{-1}\left(V^{t}\right)^{-1} D^{t}$. In particular, it is given by exponentiation as in equation (6); for $\zeta \in U(1)^{r}$,

$$
\zeta \mapsto \zeta^{U^{t}}=\left(\zeta^{U_{1}^{t}}, \cdots, \zeta^{U_{r}^{t}}\right)
$$

A standard diagram chase shows that the composition is independent of the chosen lift. That $U^{t}$ is an isomorphism follows immediately from the fact that $U$ is invertible over the integers. Furthermore, the induced action on $\bigoplus_{a=1}^{r} \mathbb{Z}_{d_{a}} \subset U(1)^{r}$ is the desired isomorphism.

The Smith normal form may be easily computed by employing, for example, the smithNormalForm() command in Macaulay2 GS. Now that we have established that the stabilizer is a finite abelian group, we show that the quotient is in fact an orbifold of $\mathbb{C}^{n}$ by this group.

Theorem 3.16. In a Landau-Ginzburg phase the quotient $X \cong \mathbb{C}^{n} / \Gamma$.

Proof. Since $R$ is full rank, for all $(x, y) \in \mathbb{C}^{N} \backslash E$, there exists a $z \in\left(\mathbb{C}^{*}\right)^{r}$ such that $z^{R} x=1$. Thus, for any $[(x, y)] \in X$,

$$
[(x, y)]=\left[\left(1, y^{\prime}\right)\right]
$$


Furthermore, since $\Gamma \subset\left(\mathbb{C}^{*}\right)^{r}$ is the stabilizer of the $x$ 's, $[(1, y)]=\left[\left(1, \gamma^{S^{t}} y\right)\right]$ for all $\gamma \in \Gamma$ and $[(1, y)] \in X$. It follows immediately that the map

$$
\begin{aligned}
X & \rightarrow \mathbb{C}^{n} / \Gamma \\
{[(1, y)] } & \mapsto[y]
\end{aligned}
$$

is an isomorphism.

Remark 3.17. As indicated above, the action of $\Gamma$ on $\mathbb{C}^{n}$ is specified by $S$ as in equation (13) and the action of the presentation $\bigoplus_{a=1}^{r} \mathbb{Z}_{d_{a}}$ of $\Gamma$ is given by

$$
\zeta \mapsto\left(\zeta^{U^{t}}\right)^{S^{t}}
$$

with $\zeta^{U^{t}}$ as in equation (14).

A natural question to ask is whether or not the affine phase is unique; that is, if there are two affine phases in the secondary fan, are the resulting quotients isomorphic? The answer is affirmative, as the following theorem shows.

Theorem 3.18. Affine quotients are unique up to unique isomorphism.

Proof. First, we note that the ring of regular functions for any quotient $X_{s}$ is independent of phase in which $s$ lies. Rational functions on any toric variety are spanned by characters on the torus, and a character is regular iff it vanishes along each toric divisor. As the order of vanishing along a divisor is given by the entries of the map $A$ in Equation 4 , the ring of regular functions depends only on $A$. Since the quotient $X_{s}$ is an affine variety whenever $s$ is an affine stable point, the result follows immediately.

As we will see in Example 4.4, the finite groups may differ across affine phases, but they yield the same action on $\mathbb{C}^{n}$.

\section{EXAMPLES}

The Herbst Criterion allows ready implementation, whether by hand or as part of a computer program. Typically, one restricts attention to the map $\mathbb{R}^{N} \rightarrow \operatorname{Im} Q$. In this case, Theorem 3.11 says that

Remark 4.1. An affine stable point exists iff there exists a non-zero maximal minor of $Q$ (arising say, from a submatrix $R$ ) such that entries of $R^{-1} Q$ away from $R$ are non-positive. Here $R$ is inverted over $\mathbb{Q}$.

As in 3.3 we may then order the columns of $Q$ so that $Q=(R S), R^{-1} S$ has non-positive rational entries, and the LG phase is Cone $(R)$. This follows from the 
changing basis for the D-terms in (2) to produce the form of $Q$ given in (5):

$$
\begin{aligned}
\sum_{i=1}^{N}\left(R^{-1} \cdot Q\right)_{i}^{a}\left|\phi^{i}\right|^{2} & =\left(R^{-1} \cdot r\right)^{a} \\
\sum_{i=1}^{N}(\mathbb{1} \mathbf{n})_{i}^{a}\left|\phi^{i}\right|^{2} & =s^{a} .
\end{aligned}
$$

Then, $R$ provides the basis change back to the original coordinates, so that positive $s^{a}$ leads to the cone over $R$. As in the proof of Lemma (3.7), $s^{a}>0$ for all $1 \leq a \leq r$ denotes an LG point.

A program to find submatrices of a specified charge matrix that define an LG phase is given in Appendix $\mathrm{A}$.

Physically, one would like to know which of the bosons in the theory (the Cox coordinates) obtain VEVs in the low-energy limit and which become coordinates in the LG phase. As mentioned before, in an LG phase the excluded set in $\mathbb{C}^{N}$ is a union of coordinate hyperplanes. In the notation above, these coordinates correspond to the first $r \phi$ 's. Assuming an appropriate choice for superpotential, one will have that these first $r$ coordinates obtain VEVs while the remaining $n$ become coordinates in the LG phase.

Example 4.2 (Example 1.1 redux). Consider a GLSM with charge matrix $Q=$ $(1,1,-2)$ and superpotential $W=\phi_{0}\left(\phi_{1}^{2}+\phi_{2}^{2}+\phi_{1} \phi_{2}\right)$. In the geometric phase the quotient variety is the canonical bundle of $\mathbb{P}^{1}$. The $\mathrm{D}$-term in this model is

$$
\left|\phi_{1}\right|^{2}+\left|\phi_{2}\right|^{2}-2\left|\phi_{0}\right|^{2}=r
$$

As before, one takes $R=(-2)$, so that $\mathbf{n}=\left(-\frac{1}{2},-\frac{1}{2}\right), s=-\frac{1}{2} r$, and the image of the polytope in $\mathbb{R}^{3}$ is

$$
\left|\phi_{0}\right|^{2}=s+\frac{1}{2}\left(\left|\phi_{1}\right|^{2}+\left|\phi_{2}\right|^{2}\right),
$$

Since the excluded set for positive $s$ is the coordinate hyperplane $\left\{\phi_{0}=0\right\}$, critical points of the superpotential occur only at $\phi_{1}=\phi_{2}=0$. Thus $\phi_{0}$ obtains a VEV, which may be chosen as $\left\langle\phi_{0}\right\rangle=\sqrt{s}$, while $\phi_{1}$ and $\phi_{2}$ become coordinates in the LG phase. It is furthermore clear that the Smith normal form of $R$ is (2), so that the finite group is $\mathbb{Z}_{2}$ acting as $\left(\phi_{1}, \phi_{2}\right) \mapsto\left(-\phi_{1},-\phi_{2}\right)$. 


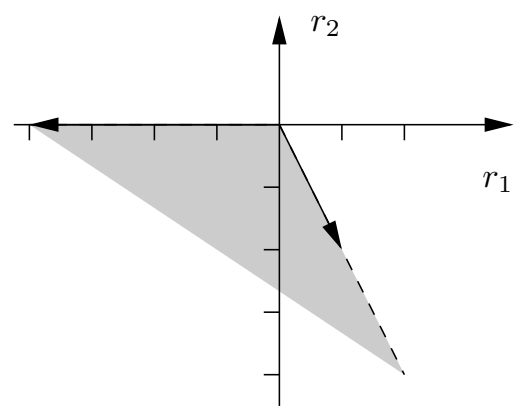

Figure 1. The Landau-Ginzburg phase for Example 4.3 .

Example 4.3 (Example 1.3 redux). In this model, we found that $Q$ in equation (1) may be written as $Q=\left(\begin{array}{ll}R & S\end{array}\right)$ with

$$
R=\left(\begin{array}{cc}
1 & -4 \\
-2 & 0
\end{array}\right) \quad \text { and } \quad S=\left(\begin{array}{ccccc}
0 & 0 & 1 & 1 & 1 \\
1 & 1 & 0 & 0 & 0
\end{array}\right)
$$

so the LG phase is given by Cone $(R)$ for appropriate choice of superpotential. See Figure 1, and compare with Figure 2 of [MRP95] (up to quantum corrections). It is easy to check that the Smith normal form of $R$ is

$$
D=\mathrm{UR} \mathrm{V}=\left(\begin{array}{ll}
8 & 0 \\
0 & 1
\end{array}\right)=\left(\begin{array}{ll}
2 & 1 \\
1 & 1
\end{array}\right)\left(\begin{array}{cc}
1 & -4 \\
-2 & 0
\end{array}\right)\left(\begin{array}{cc}
4 & -1 \\
-1 & 0
\end{array}\right),
$$

so that $\Gamma \cong \mathbb{Z}_{8} \times \mathbb{Z}_{1} \subset U(1)^{2}$ and $X=\mathbb{C}^{5} / \mathbb{Z}_{8}$. To find the action of $\mathbb{Z}_{8}$ on $\mathbb{C}^{5}$, let $\left(\zeta_{1}, \zeta_{2}\right)$ be generators of $\mathbb{Z}_{8} \times \mathbb{Z}_{1}$ so that

$$
\begin{aligned}
\zeta & \mapsto \zeta^{U_{j}^{t}} \\
\left(\zeta_{1}, \zeta_{2}=1\right) & \mapsto\left(\zeta_{1}^{2} \zeta_{2}, \zeta_{1} \zeta_{2}\right)=\left(\zeta_{1}^{2}, \zeta_{1}\right),
\end{aligned}
$$

and the action on coordinates is determined by $\mathrm{S}$ as:

$$
\left(\phi_{1}, \phi_{2}, \phi_{3}, \phi_{4}, \phi_{5}\right) \stackrel{\left(\zeta^{U^{t}}\right)^{S^{t}}}{\longmapsto}\left(\zeta_{1} \phi_{1}, \zeta_{1} \phi_{2}, \zeta_{1}^{2} \phi_{3}, \zeta_{1}^{2} \phi_{4}, \zeta_{1}^{2} \phi_{5}\right) .
$$

Example 4.4. Consider the charge matrix

$$
Q=\left(\begin{array}{cccccc}
0 & 1 & 1 & 1 & 1 & -4 \\
1 & 0 & 0 & 0 & -2 & 0
\end{array}\right) .
$$

There are two cones of the secondary fan containing affine stable points:

$$
\text { Cone }((0,1),(-4,0)) \quad \text { and } \quad \operatorname{Cone}((1,-2),(-4,0)) \text {. }
$$


One can easily check that the finite groups are $\mathbb{Z}_{4}$ and $\mathbb{Z}_{8}$, respectively, but both have $\mathbb{Z}_{4}$ actions on $\mathbb{C}^{6}$.

\section{Appendix A. Algorithm Implementation}

The following MathematicaWRI08 program takes a full-rank matrix of charges $Q$, and returns a list of the Landau-Ginzburg phases. Each phase is presented as the submatrix $R$ of $Q$ as in Remark 4.1 along with the column numbers of $Q$ from which $R$ was obtained.

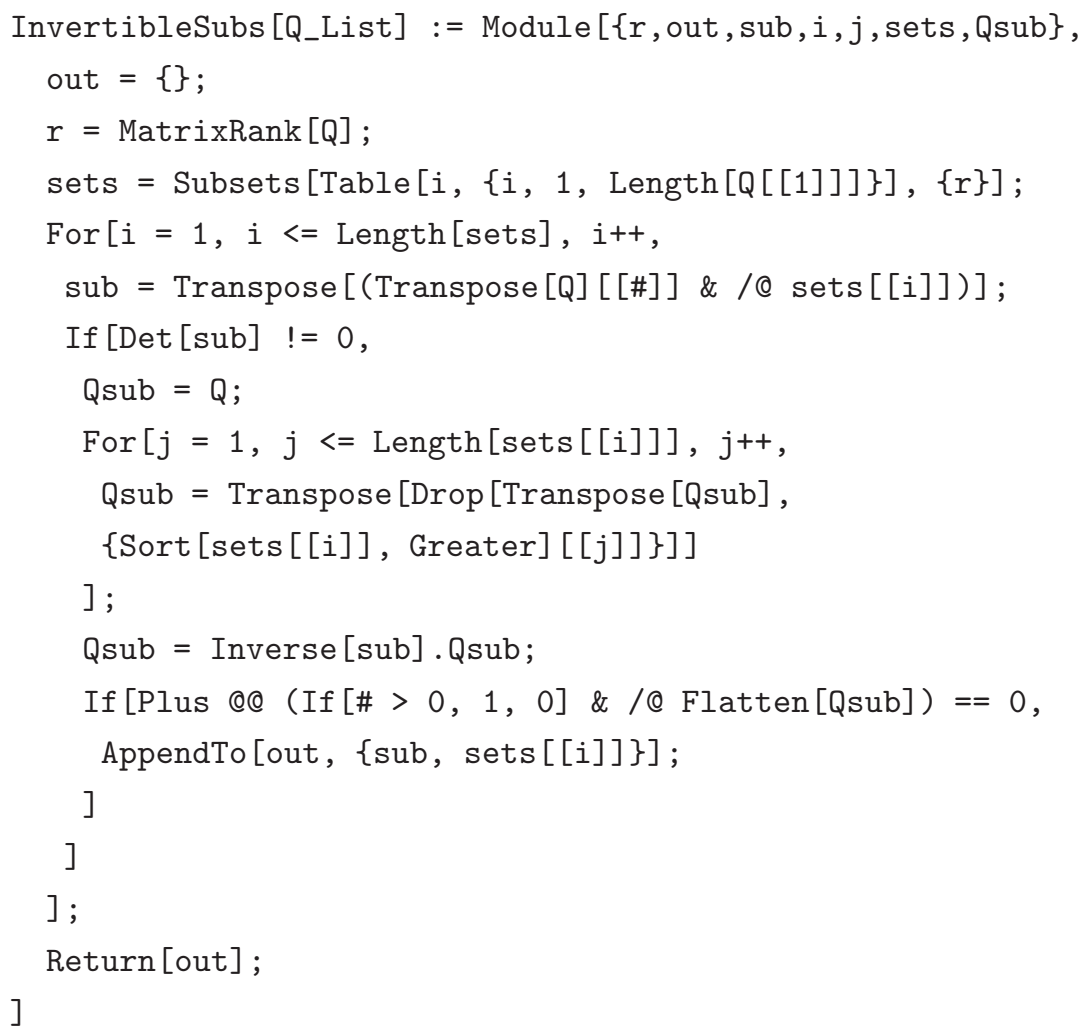

\section{REFERENCES}

[ADE06] Allan Adams, Jacques Distler, and Morten Ernebjerg. Topological heterotic rings. Adv. Theor. Math. Phys., 10:657-682, 2006, hep-th/0506263.

[Aud00] Michèle Audin. The topology of torus actions on symplectic manifolds. Progress in Mathematics. Birkhäuser, 2000.

[BFS90] Louis J. Billera, Paul Filliman, and Bernd Sturmfels. Constructions and complexity of secondary polytopes. Adv. Math., 83(2):155-179, 1990.

[CK00] D. A. Cox and S. Katz. Mirror symmetry and algebraic geometry. American Mathematical Society, 2000. Providence, USA 469 p. 
[FJR07a] Huijun Fan, Tyler J. Jarvis, and Yongbin Ruan. The Witten equation and its virtual fundamental cycle. 2007, 0712.4025.

[FJR07b] Huijun Fan, Tyler J. Jarvis, and Yongbin Ruan. The Witten equation, mirror symmetry and quantum singularity theory. 2007, 0712.4021.

[Ful93] William Fulton. Introduction to toric varieties. Princeton University Press, Princeton, 1993.

[GK07] Josh Guffin and Sheldon Katz. Deformed quantum cohomology and (0,2) mirror symmetry. 2007, arXiv:0710.2354 [hep-th].

[GS] Daniel R. Grayson and Michael E. Stillman. Macaulay2, a software system for research in algebraic geometry. Available at http://www.math.uiuc.edu/Macaulay2/

[Her09] Manfred Herbst. Private communication. 2009.

[KS06] Sheldon H. Katz and Eric Sharpe. Notes on certain $(0,2)$ correlation functions. Commun. Math. Phys., 262:611-644, 2006, hep-th/0406226.

[Mel09] Ilarion V. Melnikov. (0,2) Landau-Ginzburg Models and Residues. JHEP, 09:118, 2009, 0902.3908 .

[MM07] Jock McOrist and Ilarion V. Melnikov. Half-twisted correlators from the Coulomb branch. 2007, arXiv:0712.3272 [hep-th].

[MRP95] David R. Morrison and M. Ronen Plesser. Summing the instantons: Quantum cohomology and mirror symmetry in toric varieties. Nucl. Phys., B440:279-354, 1995, hep-th/9412236.

[Wit93] Edward Witten. Phases of $\mathrm{N}=2$ theories in two dimensions. Nucl. Phys., B403:159-222, 1993, hep-th/9301042.

[WRI08] Wolfram Research Inc. Mathematica edition: Version 7.0, 2008.

${ }^{\dagger}$ Department of Mathematics, University of Pennsylvania

E-mail address: pclarke@math.upenn.edu

‡Department of Mathematics, University of Pennsylvania

E-mail address: guffin@math.upenn.edu 